\title{
CLASSIFICATIONS OF NONNEGATIVE MATRICES USING DIAGONAL EQUIVALENCE*
}

\author{
DANIEL HERSHKOWITZ†, URIEL G. ROTHBLUM $\neq$, AND HANS SCHNEIDER§
}

\begin{abstract}
This article studies matrices $A$ that are positively diagonally equivalent to matrices that, for given positive vectors $u, v, r$, and $c$, map $u$ into $r$, and where $A^{\top}$ map $v$ into $c$. The problem is reduced to scaling a matrix for given row sums and column sums, and applying known results for the latter. Further classifications that use these results are investigated.
\end{abstract}

Key words. diagonal equivalence, nonnegative matrices, classification of nonnegative matrices

AMS(MOS) subject classifications. 65F35, 15A21, 15A48

1. Introduction. The problem of examining matrices that map a given $n$-dimensional vector into a given $m$-dimensional vector underlines many important issues in linear algebra. For example, the assertion that the row sums and/or the column sums of a matrix $A$ are given by vectors $r$ and $c$, respectively, means that $A$ maps $e$ into $r$ and $/$ or that $A^{\mathrm{T}}$ maps $e$ into $c$, where $e$ denotes the vector of appropriate dimension all of whose coordinates are 1. Also, the statement that a square matrix $A$ has a right eigenvector $u$ and a left eigenvector $v$ corresponding to a nonzero eigenvalue $\lambda$, means that $A / \lambda$ maps $u$ into $u$ and that $A^{\mathrm{T}} / \lambda$ maps $v$ into $v$. Another example is the statement that the null space of a matrix $A$ contains the vector $x$, which means that $A$ maps $x$ into the zero vector.

The purpose of this paper is to study matrices that are positively diagonally equivalent to nonnegative matrices $A$ that map $u$ into $r$, and where $A^{T}$ map $v$ into $c$ for given positive vectors $u, v, r$, and $c$. We show that, in general, the set of such matrices can be represented as the set of matrices that are positively diagonally equivalent to nonnegative matrices having prespecified row sums and column sums. We then use a known characterization of the latter class to obtain a characterization of the former class. We also characterize matrices in the intersection, as well as in the union of these classes, over all possible choices of the vectors $u, v, r$, and $c$ for which these sets are nonempty. We also obtain a special characterization for the eigenvector problem, where $m=n, u=r$, and $v=c$.

\section{Notation and definitions.}

Notation 2.1. Let $m$ and $n$ be positive integers. We denote by

$\langle n\rangle$, the set $\{1,2, \cdots, n\}$;

$R_{+0}^{\min }$, the set of all nonnegative $m \times n$ matrices;

$R_{+}^{\mathrm{D}}$, the set of all positive $n \times 1$ column vectors;

$e_{n}$, the $n \times 1$ column vector all of whose components are 1 .

Notation 2.2. For a set $\alpha$ we denote by $|\alpha|$ the cardinality of $\alpha$.

Notation 2.3. Let $A$ be an $m \times n$ matrix and let $\alpha$ and $\beta$ be nonempty subsets of $\langle m\rangle$ and $\langle n\rangle$, respectively. We denote by $A[\alpha \mid \beta]$ the submatrix of $A$ whose rows and

" Received by the editors August 10, 1987; accepted for publication (in revised form) January 13, 1988. The research of the first and second authors was supported by grant 85-00153 from the United States-Israel Binational Science Foundation (BSF), Jerusalem, Israel. The second author's research was also supported by National Science Foundation grants DMS-852152I and ECSE-18971. The third author's research was supported by National Science Foundation grant ECS-83-10213.

† Mathematics Department, Technion-Israel Institute of Technology, Haifa 32000, Israel.

\# Faculty of Industrial Engineering and Management, Technion-Israel lnstitute of Technology, Haifa 32000 , Israel.

$\S$ Mathematics Department, University of Wisconsin, Madison, Wisconsin 53706. 
columns are indexed by the elements of $\alpha$ and $\beta$, respectively, in their natural order. Also, we denote by $\alpha^{\prime}$ and $\beta^{\prime}$ the sets $\langle n\rangle \backslash \alpha$ and $\langle n\rangle \backslash \beta$, respectively.

Notation 2.4. Let $x$ be an $n \times 1$ column vector and let $\alpha \subseteq\langle n\rangle$. We denote by $x_{\alpha}$ the subvector of $x$ whose coordinates are indexed by the elements of $\alpha$.

Notation 2.5. Let $m$ and $n$ be positive integers, let $u, c \in R_{+}^{\mathrm{r}}$, and let $v, r \in R_{+}^{\mathrm{m}}$. We denote

$$
\begin{aligned}
& F_{m n}(u, v, r, c)=\left\{A \in R_{+0}^{\mathrm{ma}}: A u=r, v^{\mathbf{T}} A=c^{\mathbf{T}}\right\}, \\
& S_{m n}(r, c)=F_{m n}\left(e_{n}, e_{m}, r, c\right) .
\end{aligned}
$$

In the case that $m=n$ we denote

$$
E_{n n}(u, v)=F_{n n}(u, v, u, v) \text {. }
$$

Remark 2.6. Observe that $S_{m n}(r, c)$ is the set of all $m \times n$ nonnegative matrices with row sums $r_{1}, \cdots, r_{m}$ and column sums $c_{1}, \cdots, c_{n}$. The set $E_{n n}(u, v)$ consists of all $n \times n$ nonnegative matrices with eigenvalue 1 , where $u$ and $v$ are the corresponding right and left eigenvectors.

Notation 2.7. Let $u$ be a vector. We denote by $U$ the diagonal matrix whose diagonal elements are $u_{1}, \cdots, u_{n}$. Similar relations hold between $v, r, c$ and $V, R, C$ respectively.

DEFINITION 2.8. A diagonal matrix is said to be positive diagonal if it has positive diagonal elements.

DEFINITION 2.9. Let $A$ and $B$ be $m \times n$ matrices. We say that $A$ and $B$ are positively diagonally equivalent if there exists positive diagonal matrices $D \in R_{+0}^{\mathrm{mm}}$ and $E \in R_{+0}^{\mathrm{an}}$ such that $A=D B E$.

Notation 2.10. Let $u, c \in R_{+}^{\mathrm{n}}$ and let $v, r \in R_{+}^{\mathrm{m}}$. We denote the set of all $B \in R_{+0}^{m n}$ such that $B$ is positively diagonally equivalent to some $A \in F_{m n}(u, v, r, c)$ by $F_{m n}^{*}(u, v, r, c)$. Also, we use the following notation:

$$
S_{m n}^{*}(r, c)=F_{m n}^{*}\left(e_{n}, e_{m}, r, c\right) \text {. }
$$

and in the case that $m=n$

$$
E_{n n}^{*}(u, v) \equiv F_{n n}^{*}(u, v, u, v) \text {. }
$$

Notation 2.11. Let $A$ and $B$ be $m \times n$ matrices. We denote by $A \circ B$ the Hadamard product of $A$ and $B$, viz., the $m \times n$ matrix $C$ such that $c_{i j}=a_{i j} b_{i j}, i \in\langle m\rangle, j \in\langle n\rangle$. In particular, this notation applies when $A$ and $B$ are vectors. Obviously, the Hadamard product is commutative.

DEFINITION 2.12. An $m \times n$ matrix $A$ is said to be chainable if it has no zero row or column, and if for every pair of nonempty proper subsets $\alpha$ and $\beta$ of $\langle m\rangle$ and $\langle n\rangle$, respectively, $A[\alpha \mid \beta]=0$ implies $A\left[\alpha^{\prime} \mid \beta^{\prime}\right] \neq 0$.

DEFINITION 2.13. Let $m$ and $n$ be positive integers, let $\alpha_{1}, \cdots, \alpha_{p}$ be nonempty pairwise disjoint subsets of $\langle m\rangle$ such that $\cup_{i=1}^{p} \alpha_{i}=\langle m\rangle$, and let $\beta_{1}, \cdots, \beta_{p}$ be nonempty pairwise disjoint subsets of $\langle n\rangle$ such that $\cup_{i=1}^{p} \beta_{i}=\langle n\rangle$. An $m \times n$ matrix $A$ is said to be a (rectangular) direct sum of $A\left[\alpha_{1} \mid \beta_{1}\right], \cdots, A\left[\alpha_{p} \mid \beta_{p}\right]$ if $A\left[\alpha_{i} \mid \beta_{j}\right]=$ 0 for all $i, j \in\langle p\rangle, i \neq j$.

We comment that every rectangular matrix having no zero row or zero column is a (rectangular) direct sum of chainable matrices $A\left[\alpha_{i}, \beta_{i}\right]$ for some sets $\alpha_{1}, \cdots, \alpha_{p}$ that partition $\langle m\rangle$, and for sets $\beta_{1}, \cdots, \beta_{p}$ that partition $\langle n\rangle$.

3. The classes $F_{\text {ma }}^{*}(u, v, r, c), S_{\text {min }}^{*}(r, c)$, and $E_{\mathrm{an}}^{*}(u, v)$.

LEMMA 3.1. Let $A \in R_{+0}^{\mathrm{mm}}$, let $u, c \in R_{+}^{\mathrm{a}}$ and let $v, r \in R_{+}^{\mathrm{m}}$. Then $A \in F_{m n}(u, v, r, c)$ if and only if $V A U \in S_{m n}(r \cdot v, c \cdot u)$. 
Proof. The statement $A \in F_{m n}(u, v, r, c)$ means

$$
A U e_{n}=R e_{m}, \quad e_{m}^{\mathrm{T}} V A=e_{n}^{\mathrm{T}} C,
$$

while the statement $V A U \in S_{m n}(r \circ v, c \circ u)$ means

$$
V A U e_{n}=V R e_{m}, \quad e_{m}^{\mathrm{T}} V A U=e_{n}^{\mathrm{T}} C U .
$$

The equivalence of (3.2) and (3.3) is clear.

COROLlaRY 3.4. Let $u, c \in R_{+}^{\mathrm{n}}$ and let $v, r \in R_{+}^{\mathrm{m}}$. If $F_{m n}(u, v, r, c)$ is nonempty then $v^{\mathrm{T}} r=c^{\mathrm{T}} u$.

Proof. The result follows directly from Lemma 3.1 and the corresponding standard result concerning the transportation problem.

COROllary 3.5. Let $A \in R_{+0}^{\mathrm{mn}}$, let $u, c \in R_{+}^{\mathrm{n}}$, and let $v, r \in R_{+}^{\mathrm{m}}$. Then $A \in$ $F_{m n}^{*}(u, v, r, c)$ if and only if $A \in S_{m n}^{*}(r \cdot v, c \cdot u)$.

COROLlaRY 3.6. Let $A \in R_{+0}^{\text {nn }}$ and let $u, v \in R_{+}^{\mathrm{n}}$. Then $A \in E_{n n}^{*}(u, v)$ if and only if $A \in S_{m n}^{*}(u \cdot v, u \cdot v)$.

The following theorem is proved in [3] as Theorems 3.9 and 4.1. We state it here in a slightly different way.

THEOREM 3.7. Let $A \in R_{+0}^{\mathrm{mn}}$ have no zero row or zero column, let $c \in R_{+}^{\mathrm{n}}$, and let $r \in R_{+}^{\mathrm{m}}$. Then we have the following:

(i) When $A$ is chainable, then $A \in S_{m n}^{*}(r, c)$ if and only if for every pair of nonempty proper subsets $\alpha$ and $\beta$ of $\langle m\rangle$ and $\langle n\rangle$ we have

$$
A[\alpha \mid \hat{\beta}]=0 \text { and } A\left[\alpha^{\prime} \mid \beta^{\prime}\right] \neq 0 \Rightarrow \sum_{i \in \alpha} r_{i}<\sum_{i \in \beta^{\prime}} c_{i} .
$$

In this case, there exist unique (up to scalar multiplication) positive diagonal matrices $D$ and $E$ such that $D A E \in S_{m n}(r, c)$.

(ii) $A \in S_{m n}^{*}(r, c)$ if and only if $A$ is a direct sum of chainable matrices $A\left[\alpha_{i} \mid \beta_{i}\right]$, $i=1, \cdots, p$, such that

$$
A\left[\alpha_{i} \mid \beta_{i}\right] \in S_{\left|\alpha_{i}\right| \beta_{i} \mid}^{*}\left(r_{\alpha_{i}}, c_{\beta_{i}}\right), \quad i \in\langle p\rangle .
$$

(iii) If $A \in S_{m n}^{*}(r, c)$ then there exists a unique matrix in $S_{m n}(r, c)$ which is positively diagonally equivalent to $A$.

Remark 3.8. Statement (iii) in Theorem 3.7 follows immediately from statement (ii). Observe that in statement (iii) we do not assert uniqueness of the positive diagonal matrices $D$ and $E$ such that $D A E \in S_{m n}(r, c)$, but the uniqueness of the matrix $D A E$.

We now use our results in order to generalize Theorem 3.7. The following result also generalizes Theorem 3.10 of [1] and Theorem 3.2 of [2].

THEOREM 3.9. Let $A \in R_{+0}^{\mathrm{m}}$ have no zero row or zero column, let $u, c \in R_{+}^{\mathbf{n}}$, and let $v, r \in R_{+}^{\mathrm{m}}$. Then we have the following:

(i) When $A$ is chainable then $A \in F_{m n}^{*}(u, v, r, c)$ if and only if for every pair of nonempty proper subsets $\alpha$ and $\beta$ of $\langle m\rangle$ and $\langle n\rangle$ we have

$$
A[\alpha \mid \beta]=0 \text { and } A\left[\alpha^{\prime} \mid \beta^{\prime}\right] \neq 0 \Rightarrow v_{\alpha}^{\top} r_{\alpha}<c_{\beta}^{\top}, u_{\beta^{\prime}} .
$$

In this case, there exist unique (up to scalar multiplication) positive diagonal matrices $D$ and $E$ such that $D A E \in F_{m n}(u, v, r, c)$.

(ii) $A \in F_{m n}^{*}(u, v, r, c)$ if and only if $A$ is a direct sum of chainable matrices $A\left[\alpha_{i} \mid \beta_{i}\right], i=1, \cdots, p$, such that

$$
A\left[\alpha_{i} \mid \beta_{i}\right] \in F_{\left|\alpha_{\alpha}\right| \beta_{i} \mid}^{*}\left(u_{\beta_{i}}, v_{\alpha_{i}}, r_{\alpha_{i}}, c_{\beta_{i}}\right), \quad i \in\langle p\rangle .
$$


(iii) If $A \in F_{m n}^{*}(u, v, r, c)$ then there exists a unique matrix in $F_{m n}(u, v, r, c)$ which is positively diagonally equivalent to $A$.

Proof. The assertion follows directly from Corollary 3.5 and Theorem 3.7.

In view of Corollary 3.4, statements (i) and (ii) of Theorem 3.9 can be combined and restated as Theorem 3.10.

THEOREM 3.10. Let $A \in R_{+0}^{\mathrm{ma}}$, have no zero row or zero column, let $u, c \in R_{+}^{\text {, }}$, and let $v, r \in R_{+}^{\mathrm{m}}$. Then $A \in F_{m n}^{*}(u, v, r, c)$ if and only if for every pair of nonempty proper subsets $\alpha$ and $\beta$ of $\langle m\rangle$ and $\langle n\rangle$, respectively, we have

$$
\begin{array}{lll}
A[\alpha \mid \beta]=0 & \text { and } & A\left[\alpha^{\prime} \mid \beta^{\prime}\right] \neq 0 \Rightarrow v_{\alpha}^{\mathbf{T}} r_{\alpha}<c_{\beta^{\prime}}^{\mathbf{T}}, u_{\beta^{\prime}}, \\
A[\alpha \mid \beta]=0 & \text { and } & A\left[\alpha^{\prime} \mid \beta^{\prime}\right]=0 \Rightarrow v_{\alpha}^{\mathbf{T}} r_{\alpha}=c_{\beta^{\prime}}^{\mathbf{T}} u_{\beta^{\prime}} .
\end{array}
$$

4. The classes $\cap F_{\mathrm{min}}^{*}, \cup F_{\mathrm{min}}^{*}, \cap S_{\mathrm{mn}}^{*}, \cup S_{\mathrm{mn}}^{*}, \cap E_{\mathrm{mn}}^{*}$, and $\cup E_{\mathrm{mn}}^{*}$.

Notation 4.1. Let $m$ and $n$ be positive integers. We denote the following:

$$
\begin{aligned}
& \cap F_{m n}^{*}=\bigcap_{\substack{\mu, c \in R^{*} \\
v, r \in R_{+}^{ \pm} \\
u^{2} c=v^{\frac{1}{r}} r}} F_{m n}^{*}(u, v, r, c), \\
& \cup F_{m n}^{*}=\bigcup_{\substack{u, c \in R^{\ddagger} \\
v, r \in R_{+}^{\ddagger}}} F_{m n}^{*}(u, v, r, c) \text {, } \\
& \cap S_{m n}^{*}=\bigcap_{\substack{c \in R_{+}^{q} \\
r \in R_{+}^{+} \\
e_{n}^{\top} c=e_{m}^{T}}} S_{m n}^{*}(r, c), \\
& \cup S_{m n}^{*}=\bigcup_{\substack{c \in R_{\ddagger}^{*} \\
r \in R_{\ddagger}^{\prime}}} S_{m n}^{*}(r, c) \text {, } \\
& \cap E_{n n}^{*}=\bigcap_{u, v \in R_{+}^{*}} E_{n n}^{*}(u, v) \text {, } \\
& \cup E_{n n}^{*}=\bigcup_{u, v \in R_{+}^{\ddagger}} E_{n n}^{*}(u, v) .
\end{aligned}
$$

THEOREM 4.2. Let $A \in R_{+0}^{\mathrm{m}}$. Then we have the following:

(i) $A \in \cap F_{m n}^{*}$ if and only if $A$ has no zero entries.

(ii) $A \in \cup F_{m n}^{*} \backslash \cap F_{m n}^{*}$ if and only if $A$ has at least one zero entry but there is no zero row or zero column in $A$.

(iii) $A \notin \cup F_{m n}^{*}$ if and only if $A$ has at least one zero row or zero column.

Proof. (i) Let $A \in R_{+0}^{\mathrm{mn}}$. If $A$ has no zero entries then Theorem 3.10 immediately implies that $A \in \cap F_{m n}^{*}$. Conversely, we show that if $a_{i j}=0$ for some $i \in\langle m\rangle$ and $j \in$ $\langle n\rangle$, then $A \notin \cap F_{m n}^{*}$. We choose $u, c \in R_{+}^{\mathfrak{n}}$ with $u_{j} c_{j}=\frac{3}{3}$ and $u^{\mathrm{T}} c=1$, and $v, r \in R_{+}^{\mathrm{m}}$ with $v_{i} r_{i}=3$ and $v^{\mathrm{T}} r=1$. Then for $\alpha=\{i\}$ and $\beta=\{j\}$ we have that

$$
v_{\alpha}^{\mathbf{T}} r_{\alpha}=\frac{2}{3}>\frac{1}{3}=u^{\mathbf{T}} c-u_{j} c_{j}=u_{\beta^{\prime}}^{\mathbf{T}} c_{\beta^{\prime}} .
$$

Since $A[\alpha \mid \beta]=0$ it now follows from Theorem 3.10 that $A \notin F_{m n}^{*}(u, v, r, c)$.

(ii) Let $A \in \cup F_{m n}^{*} \backslash \cap F_{m n}^{*}$. By (i), $A$ has at least one zero entry. Since $A$ belongs to some $F_{m n}^{*}(u, v, r, c)$, where $u, v, r, c$ are strictly positive vectors, it follows that $A$ has neither a zero row nor a zero column. Conversely, if $A$ has a zero entry but no zero row or zero column, then by (i), $A \notin \cap F_{m n}^{*}$. Moreover, $A \in F_{m n}\left(e_{n}, e_{m}, r, c\right)$, where $r$ and $c$ are, respectively, the strictly positive vectors of row sums and column sums of $A$.

(iii) This equivalence follows directly from (i) and (ii). 
The next theorem shows that the classifications with respect to $S_{m n}^{*}$ and $F_{m n}^{*}$ coincide.

THEOREM 4.3. We have $\cap S_{m n}^{*}=\cap F_{m n}^{*}$ and $\cup S_{m n}^{*}=\cup F_{m n}^{*}$.

Proof. Trivially, $\cap F_{m n}^{*} \subseteq \cap S_{m n}^{*}$ and $\cup S_{m n}^{*} \subseteq \cup F_{m n}^{*}$. The reverse inclusions follow immediately from Corollary 3.5 .

Recall that a square matrix is said to be completely reducible if for some permutation matrix $P$, the matrix $P A P^{\mathrm{T}}$ is a direct sum of irreducible matrices.

THEOREM 4.4. Let $A \in R_{+0}^{\text {nn }}$. Then we have the following:

(i) $A \in \cap E_{n n}^{*}$ if and only if $A$ is completely reducible and the diagonal elements of $A$ are positive.

(ii) $A \in \cup E_{n n}^{*} \backslash \cap E_{n n}^{*}$ if and only if $A$ is completely reducible, $a_{i i}=0$ for some $i \in$ $\langle n\rangle$, and $A$ has no zero row or zero column.

(iii) $A \notin \cup E_{n n}^{*}$ if and only if either $A$ is not completely reducible or $A$ has a zero row or zero column.

Proof. Since the conditions in (i)-(iii) are mutually exclusive and collectively exhaustive, it is enough to prove the "if" part in each of the three assertions.

(i) Suppose that $A$ is completely reducible with positive diagonal elements. Let $u, v \in R_{+}^{\mathrm{a}}$ and let $\alpha$ and $\beta$ be nonempty proper subsets of $\langle n\rangle$. Suppose that

$$
A[\alpha \mid \beta]=0 \text {. }
$$

Also, suppose that

$$
A\left[\alpha^{\prime} \mid \beta^{\prime}\right] \neq 0 \text {. }
$$

Since $A$ has positive diagonal elements it follows from (4.5) that $\alpha \cap \beta=\varnothing$, i.e., $\alpha \subseteq$ $\beta^{\prime}$. We claim that $\alpha$ is a proper subset of $\beta^{\prime}$. Suppose to the contrary that $\alpha=\beta^{\prime}$. Then (4.5) and (4.6) imply that $A\left[\beta^{\prime} \mid \beta\right]=0$ and $A\left[\beta \mid \beta^{\prime}\right] \neq 0$, contradicting the assumption that $A$ is completely reducible. Thus, $\gamma=\alpha \cup \beta$ is a proper subset of $\langle n\rangle$ and, since $\alpha \cap \beta=\varnothing$, we have

$$
v_{\alpha}^{\mathrm{T}} u_{\alpha}+v_{\beta}^{\mathrm{T}} u_{\beta}=v_{\gamma}^{\mathrm{T}} u_{\gamma}<v^{\mathrm{T}} u
$$

implying that

$$
v_{\alpha}^{\mathrm{T}} u_{\alpha}<v^{\mathrm{T}} u-v_{\beta}^{\mathrm{T}} u_{\beta}=v_{\beta}^{\mathrm{T}} u_{\beta^{\prime}}
$$

Now suppose that (4.5) holds and that

$$
A\left[\alpha^{\prime} \mid \beta^{\prime}\right]=0 \text {. }
$$

As before, (4.5) implies that $\alpha \subseteq \beta^{\prime}$. Similarly, (4.9) implies that $\alpha^{\prime} \subseteq \beta$, i.e., $\beta^{\prime} \subseteq \alpha$. So, $\alpha=\beta^{\prime}$, and hence $v_{\alpha}^{\mathrm{T}} u_{\alpha}=v_{\beta}^{\mathrm{T}} u_{\beta^{\prime}}$. It now follows from Theorem 3.10 that

$$
A \in F_{m n}^{*}(u, v, u, v)=E_{n n}^{*}(u, v) \text {. }
$$

(ii) Suppose that $A$ is completely reducible, that $a_{i j}=0$ for some $i \in\langle n\rangle$, and that $A$ has no zero row or zero column. We choose $u, v \in R_{+}^{\mathrm{p}}$ with $v_{i} u_{i}=\boldsymbol{z}$ and $v^{\mathbf{T}} u=1$. Then for $\alpha=\beta=\{i\}$ we have

$$
v_{\alpha}^{\mathbf{T}} u_{\alpha}=\frac{2}{3}>\frac{1}{3}=v^{\mathbf{T}} u-v_{i} u_{i}=v_{\beta}^{\mathbf{T}}, u_{\beta^{\prime}} .
$$

Therefore, by Theorem 3.10 and Notation 2.10 we have $A \notin E_{n n}^{*}(u, v)$. We now have to show that $A \in \cup E_{n n}^{*}$. Since $A$ is completely reducible, it follows that $A$ is a direct sum of irreducible matrices. Furthermore, since $A$ has no zero row or zero column, each of these irreducible matrices is nonzero and thus has a positive spectral radius. It now follows that we can find a matrix $B$ which is positively diagonally equivalent to $A$, where 
$B$ is a direct sum of irreducible matrices with spectral radii 1. By the Perron-Frobenius theory for nonnegative matrices it follows that for some $u, v \in R_{+}^{*}$ we have $B u=u$ and $v^{\mathrm{T}}=v^{\mathrm{T}} B$, i.e., $B \in E_{n n}(u, v)$. Hence $A \in E_{n n}^{*}(u, v) \subseteq \cup E_{n n}^{*}$.

(iii) In the case that $A$ has a zero row or zero column the assertion is clear. Suppose that $A$ is not completely reducible. Then there exist nonempty subsets $\alpha$ and $\beta$ of $\langle n\rangle$ with $\alpha=\beta^{\prime}$, such that $A[\alpha \mid \beta]=0$ and $A\left[\alpha^{\prime} \mid \beta^{\prime}\right] \neq 0$. Since $\alpha=\beta^{\prime}$, for every $u, v \in$ $R_{+}^{\mathrm{n}}$ we have $v_{\alpha}^{\mathrm{T}} u_{\alpha}=v_{\beta^{\prime}}^{\mathrm{T}} u_{\beta^{\prime}}$. By Theorem 3.10 it now follows that $A \notin E_{n n}^{*}(u, v)$.

Our final observation shows that the requirement $u=v$ in $E_{n n}^{*}(u, v)$ or $r=c$ in $S_{m n}^{*}(r, c)$ does not yield new classifications. Specifically, let

$$
\begin{aligned}
& \cap E_{n}^{*}=\bigcap_{u \in R_{+}^{\ddagger}} E_{n n}^{*}(u, u), \\
& \cup E_{n}^{*}=\bigcup_{u \in R_{+}^{\sharp}} E_{n n}^{*}(u, u), \\
& \cap S_{n}^{*}=\bigcap_{r \in R_{+}^{\ddagger}} S_{n n}^{*}(r, r), \\
& \cup S_{n}^{*}=\bigcup_{r \in R_{+}^{\ddagger}} S_{n n}^{*}(r, r) .
\end{aligned}
$$

THEOREM 4.10. We have

$$
\begin{aligned}
& \cap S_{n}^{*}=\cap E_{n}^{*}=\cap E_{n n}^{*}, \\
& \cup S_{n}^{*}=\cup E_{n}^{*}=\bigcup E_{n n}^{*} .
\end{aligned}
$$

Proof. For $u \in R_{+}^{\mathrm{a}}$, let $u^{(1 / 2)}$ be the vector in $R_{+}^{\mathrm{a}}$ with $\left(u^{(1 / 2)}\right)_{i}=\left(u_{i}\right)^{1 / 2}, i=1$, $\cdots, n$. Then Corollary 3.6 shows that $S_{n n}^{*}(u, u)=E_{n n}^{*}\left(u^{(1 / 2)}, u^{(1 / 2)}\right)$, implying that $\cap E_{n}^{*} \subseteq \cap S_{n}^{*}$ and $\cup S_{n}^{*} \subseteq \cup E_{n}^{*}$. Next, the inclusions $\cap E_{n n}^{*} \subseteq \cap E_{n}^{*}$ and $\cup E_{n}^{*} \subseteq \cup E_{n n}^{*}$ are immediate, and the inclusions $\cap S_{n}^{*} \subseteq \cap E_{n n}^{*}$ and $\cup E_{n n}^{*} \subseteq \cup S_{n}^{*}$ follow directly from Corollary 3.6. Thus, the conclusions of our theorem have been established.

\section{REFERENCES}

[1] A. Berman and R. J. Plemmons, Nonnegative Matrices in the Mathematical Sciences, Academic Press, New York, 1979.

[2] S. FRIEDLAND AND S. KARLIN, Some inequalities for the spectral radius of non-negative matrices and applications, Duke Math. J., 62 (1975), pp. 459-490.

[3] M. V. MENON AND H. SCHNEIDER, The spectrum of a nonlinear operator associated with a matrix, Linear Algebra Appl., 2 (1969), pp. 321-334. 